Article

\title{
Wander of a Gaussian-Beam Wave Propagating through Kolmogorov and Non-Kolmogorov Turbulence along Laser-Satellite Communication Uplink
}

\author{
Fazhi Wang, Wenhe Du* , Qi Yuan, Daosen Liu and Shuang Feng
}

Citation: Wang, F.; Du, W.; Yuan, Q.; Liu, D.; Feng, S. Wander of a Gaussian-Beam Wave Propagating through Kolmogorov and Non-Kolmogorov Turbulence along Laser-Satellite Communication

Uplink. Atmosphere 2022, 13, 162. https://doi.org/10.3390/ atmos13020162

Academic Editors: Evgeniy A. Kopylov, Artem Yu. Shikhovtsev, Vladimir P. Lukin

Received: 17 December 2021

Accepted: 13 January 2022

Published: 20 January 2022

Publisher's Note: MDPI stays neutral with regard to jurisdictional claims in published maps and institutional affiliations.

Copyright: (c) 2022 by the authors. Licensee MDPI, Basel, Switzerland. This article is an open access article distributed under the terms and conditions of the Creative Commons Attribution (CC BY) license (https:// creativecommons.org/licenses/by/ $4.0 /)$.
College of Telecommunication and Electronic Engineering, Qiqihar University, Qiqihar 161006, China; wangfazhi2001@163.com (F.W.); foreveryuanqi@126.com (Q.Y.); atocom@yeah.net (D.L.); whduhit@qqhru.edu.cn (S.F.)

* Correspondence: atocom@163.com

\begin{abstract}
It is accepted that there exists two kinds of atmospheric turbulence in the Earth's aerosphere-Kolmogorov and non-Kolmogorov turbulence; therefore, it is important to research their combined impacts on laser-satellite communications. In this paper, the exponential power spectra of refractive-index fluctuations for non-Kolmogorov turbulence in the free troposphere and stratosphere are proposed, respectively. Based on these two spectra, using the Markov approximation, beam wander displacement variances of a Gaussian-beam wave are derived, respectively, which are valid under weak turbulent fluctuations condition. On this basis, using a three-layer altitude-dependent turbulent spectrum model for vertical/slant path, the combined influence of a three-layer atmospheric turbulence on wander of a Gaussian-beam wave as the carrier wave in laser-satellite communication is studied. This three-layer spectrum is more accurate than a two-layer model. Moreover, the variations of beam wander displacement with beam radius, zenith angles, and nominal value of the refractive-index structure parameter on the ground are estimated. The theory of optical wave propagation through non-Kolmogorov atmospheric turbulence is further enriched and a theoretical model of a three-layer atmospheric turbulence beam wander for a satellite-ground laser communication uplink is established.
\end{abstract}

Keywords: satellite laser communication; atmospheric optics; non-Kolmogorov turbulence; Kolmogorov turbulence; beam wander

\section{Introduction}

Since the 1960s, as one of the advanced satellite communication technologies, satellite laser communication technology has attracted much attention from the scientific community because it has some potential advantages, including higher data rates, larger communication capability, better anti-disturbance, low probability of intercept, less volume, less mass, lower power consumption, no restrictions for frequency use, etc. [1]. At the same time, the integration of the newly developed satellite communication technology and satellite laser communication technology can also be expected [2-4]. In the late 1990s, the United States, Europe, and Japan successively carried out verification experiments on the ground. In the first two decades of the 21st century, satellite laser communication technology has made great progress. As the representatives of space optical communication pioneer, the European Space Agency, the German Space Agency, the Japan aerospace exploration agency, the National Association of Information and Communications Technology, and the Jet Propulsion Laboratory involve comprehensive and in-depth research, and key technologies have been developed. Several sets of satellite laser communication terminals have been developed and successfully completed a number of on-orbit experiments. The space-based laser communication network with global coverage are being planned to demonstrate the huge potential of satellite laser communications and lay a solid foundation 
for the commercialization and application of satellite laser communication technology. For satellite-to-ground and ground-to-satellite links, since the Earth's atmosphere is one part of the communication channel, the atmospheric turbulence deteriorates the performance level of satellite laser communication system. To solve this problem, several experiments have been carried out to study the effects of atmospheric turbulence on satellite-ground laser communication. The results have shown that the laser uplink is more seriously affected by atmospheric turbulence than the downlink. The main performance is that the bit error rate of the uplink is much higher than that of the downlink [5-8]. In the downlink, the above 90 percent path of the laser beam is the ideal transmission environment close to vacuum, and there will be atmospheric attenuation and phase distortion until it is close to the ground. On the contrary, in the uplink, the laser beam is affected by the dense atmosphere of the troposphere as soon as it leaves the pupil. In addition to atmospheric attenuation and scintillation, the downlink is also affected seriously by the beam wander, which is equivalent to increase the pointing error of the satellite laser communication system. Up until now, the uplink of satellite-ground laser communication can only barely establish in all on-orbit experiments, and cannot guarantee the continuous symbol tracking and clock synchronization, and there is often the phenomenon of communication interruption. Therefore, it is of great significance to study the influence of atmospheric turbulence on satellite laser communication, especially on uplink.

In recent years, data from stellar scintillation measurements, airborne tether balloon temperature measurements, and ground-based radar measurements have shown turbulence deviations from Kolmogorov's model in parts of the Earth's atmosphere [9-13]. For example, Dalaudier et al. [10] measured the power spectrum of one-dimensional temperature fluctuations along the inclined path of the Earth's atmosphere by using balloons and radar. The results showed that the upper troposphere and the entire stratospheric turbulent atmosphere did not follow the Kolmogorov statistical law, and the three-dimensional power spectrum of -5 power rate was also confirmed. In theory, further development of the propagation theory of passive conservative quantity has shown that although the Kolmogorov spectrum is important, it is only part of more general behavior of passive conservative quantity propagation in turbulence. At the same time, other theoretical studies further confirm this conclusion [14-16]. For example, when the atmosphere is in a relatively stable state that are not locally isotropic, the behavior is inconsistent with the Kolmogorov turbulence statistical law [14]. Dewan [16] conducted a numerical study on the power spectrum of internal gravity waves and the results confirmed the existence of a turbulent power spectrum with a power ratio of -5 , which corresponds to internal gravity waves (IGW). IGW are anisotropic structures. Lukin et al. [17-19] suggested a hypothesis that atmospheric coherent structures were principal causes of considerable deviations of the Kolmogorov and Obukhov constants from their standard values. Turbulence observed in a coherent structure is called coherent turbulence, which is different from noncoherent Kolmogorov turbulence. Its one-dimensional spectrum has one more rapid decrease in the inertia interval and lower contributions of small-scale components. Phase fluctuations of optical radiation in coherent turbulence are weaker as compared to the Kolmogorov one (due to lack in small-scale inhomogeneities).

The combined influence of Kolmogorov and non-Kolmogorov turbulence on optical wave has been investigated along a slant or vertical path on the basis of further development of a propagation theory for optical wave in non-Kolmogorov turbulence, including beam spreading, intensity fluctuations, etc. [20,21]. These studies were carried out by establishing a two-layer turbulence model based on the early observations of atmospheric turbulence. Namely the turbulence in the earth's atmosphere is composed of Kolmogorov turbulence (spectral exponent -11/3) in the troposphere and non-Kolmogorov turbulence (spectral exponent -5) in the stratosphere. The latest measurements showed that the atmosphere turbulence in the the boundary layer obeyed the Kolmogorov theory and that the atmosphere turbulence in the free troposphere was different from one in the stratosphere, both of which exhibited non-Kolmogorov properties. The more accurate three-layer 
atmospheric turbulence model was developed. The related works also were extended from the two-layer atmospheric turbulence model to the three-layer one, including scintillation index of a plane and spherical wave at large zenith angles, propagation of electromagnetic waves, etc. [22-24]. The fluctuation conditions also gradually expanded from weak to strong fluctuations. It is well-known that beam wander can cause time-varying power fades at the receiver terminal and further deteriorates the performance of satellite laser communication system. Meanwhile, beam wander also affects the acquiring probability, acquiring time, pointing accuracy, and tracking stability of the laser communication link and then degrades the performance of satellite laser communication pointing, acquiring, and tracking subsystem. Therefore, it is significant to study the combined influence of the three-layer atmospheric turbulence on the wander of the laser beam wave as the carrier wave in laser satellite communication.

In this paper, a three-layer altitude-dependent power spectrum of refractive-index fluctuations for satellite-to-ground and ground-to-satellite links is modified, which is composed of the Kolmogorov power spectrum with an exponent value of $-11 / 3$, non-Kolmogorov ones with exponent values of $-10 / 3$ and -5 , corresponding to the boundary layer, the free troposphere, and the stratosphere in the aerosphere, respectively. Using the Markov approximation, beam wander displacement variances of a Gaussian-beam wave propagating through non-Kolmogorov turbulence in the free troposphere and the stratosphere are derived, respectively. Finally, an expression governing wander of a Gaussian-beam wave along a slant path valid in the weak fluctuations regime is presented. This work lays a scientific foundation for the establishment of an atmospheric turbulence compensation model and has certain scientific significance for improving the performance of a satellite-ground laser communications system, on-orbit experiment and practical application.

\section{Three-Layer Altitude Spectrum Model of Refractive-Index Fluctuations}

Increasing measurements of the atmosphere have shown that the turbulence statistical characteristics in the atmosphere varies with altitude. Up until now, the experimental results have shown that the boundary layer (up to $\sim 2-3 \mathrm{~km}$ ) had Kolmogorov turbulent characteristics and the free troposphere (up to $\sim 8-10 \mathrm{~km}$ ) and the stratosphere showed different non-Kolmogorov turbulent characteristics. Based on this, Zilberman et al. [24] suggested a three-layer altitude spectrum model that is more accurate, in which the first layer corresponds to the boundary layer with Kolmogorov turbulence (with spectrum exponent value of 11/3); the second one corresponds to the free troposphere with nonKolmogorov turbulence (with spectrum exponent value of 10/3), and the third layer where non-Kolmogorov turbulence spectrum has a slope 5 . The values of the refractive index structure constant for the free tropospheric turbulence and stratospheric one are given, respectively, $A(\alpha=10 / 3)=0.015$ and $A(\alpha \longrightarrow 5)=0.0024$. As mentioned above, the conclusions developed by Lukin et al. can present a reasonable theoretical basis. Since the turbulence is developed in the boundary layer and the Kolmogorov turbulence appears, the difference in the sizes of primary vortices of different coherent structures is small in the area under study and the sum of the spectra of different coherent structures is close to a Kolmogorov power dependence. On the contrary, the turbulence is undeveloped in the free troposphere and the stratosphere and coherent turbulence appears, so the difference in the sizes of primary vortices of different coherent structures is high, and the sum of the spectrum shows a rapid power decrease, corresponding to non-Kolmogorov power dependence $[17,18]$.

Here, a three-layer altitude-dependent turbulent spectrum model of refractive-index fluctuations for a vertical/slant path is proposed to describe the variations of turbulent statistical characteristics with altitudes in the aerosphere in Reference [22], which is equal to the sum of refractive-index fluctuation power spectra in the boundary layer, the free troposphere, and the stratosphere. It is shown that the turbulence is characterized by the 
conventional Kolmogorov model in the boundary layer and the refractive-index fluctuations power spectrum has the following form,

$$
\Phi_{n B}(\kappa, z)=0.033 C_{n}^{2}(z) \kappa^{-11 / 3},
$$

where $\kappa$ is the magnitude of the spatial frequency vector in units of $\mathrm{rad} / \mathrm{m}, z$ is a propagation distance that varies between $z=0$ and $z=L$, and $C_{n}^{2}(z)$ is the conventional Kolmogorov turbulent index-of-refraction structure parameter in the boundary layer that is dependent on the altitude and has units of $\mathrm{m}^{-2 / 3}$. The turbulence exhibits non-Kolmogorov characteristics in the free troposphere and the refractive-index fluctuations power spectrum has the following form,

$$
\Phi_{n F}(\kappa, z)=0.015 \widetilde{C}_{n F}^{2}(z) \kappa^{-10 / 3},
$$

where $\widetilde{C}_{n F}^{2}(z)$ is non-Kolmogorov index-of-refraction structure parameter in the free troposphere that depends on the altitude and has units of $\mathrm{m}^{-1 / 3}$. The turbulence exhibits another non-Kolmogorov characteristics in the stratosphere above them and the refractive-index fluctuations power spectrum has the following form,

$$
\Phi_{n S}(\kappa, z)=0.0024 \widetilde{C}_{n S}^{2}(z) \kappa^{-5},
$$

where $\widetilde{C}_{n S}^{2}(z)$ is non-Kolmogorov index-of-refraction structure parameter in the stratosphere that is dependent on the altitude and has units of $\mathrm{m}^{-2}$ [22].

Based on the Markov approximation [25], the only larger inhomogeneities than the scale of the beam size can contribute to the beam wander, so the exponential spectrum for the Kolmogorov turbulence in the boundary layer is used,

$$
\Phi_{n B}(\kappa, z)=0.033 C_{n}^{2}(z) \kappa^{-11 / 3}\left[1-\exp \left(-\frac{\kappa^{2}}{\kappa_{0}^{2}(h)}\right)\right],
$$

where $\kappa_{0}(z)=C_{0} / L_{0}(h)$, here, the scaling constant $C_{0}$ is chosen in the range $1 \leq C_{0} \leq 8 \pi$, here the value of $C_{0}$ is $2 \pi$, and $L_{0}(h)$ is the outer scale varying with the altitude. Here, in the same way, the outer scale factors are introduced in the refractive-index fluctuations power spectra of the free troposphere and the stratosphere,

$$
\begin{gathered}
\Phi_{n F}(\kappa, z)=0.015 \widetilde{C}_{n F}^{2}(z) \kappa^{-10 / 3}\left[1-\exp \left(-\frac{\kappa^{2}}{\kappa_{0}^{2}(h)}\right)\right], \\
\Phi_{n S}(\kappa, z)=0.0024 \widetilde{C}_{n S}^{2}(z) \kappa^{-5}\left[1-\exp \left(-\frac{\kappa^{2}}{\kappa_{0}^{2}(h)}\right)\right] .
\end{gathered}
$$

And the three-layer altitude spectrum model was developed [24]. In order to study the combined influence of the Kolmogorov turbulence and the non-Kolmogorov one on laser propagation for an uplink/downlink communication channel, based on Equations (4)-(6), a modified three-layer altitude spectrum model of refractive-index fluctuations is suggested, which has the following form,

$$
\Phi_{n}(\kappa, z)=\Phi_{n B}(\kappa, z)+\Phi_{n F}(\kappa, z)+\Phi_{n S}(\kappa, z) .
$$

Propagation along a vertical or slant path requires a $C_{n}^{2}(h)$ profile model to describe properly the varying strengths of optical turbulence as functions of altitude $h$. For the $C_{n}^{2}(h)$ profile, the most widely used Hufnagel-Valley model is chosen [1]

$$
\begin{aligned}
C_{n}^{2}(h) & =0.00594(v / 27)^{2}\left(10^{-5} h\right)^{10} \exp (-h / 1000) \\
& +2.7 \times 10^{-16} \exp (-h / 1500)+C_{n}^{2}(0) \exp (-h / 100)
\end{aligned}
$$


with the wind speed $v=21 \mathrm{~m} / \mathrm{s}$ and $C_{n}^{2}(0)=1.7 \times 10^{-14} \mathrm{~m}^{-2 / 3}$. For the $\widetilde{C}_{n F}^{2}(h)$ profile in the free troposphere, this form is taken as suggested in [24],

$$
\widetilde{C}_{n F}^{2}(h)=2.2\left(\frac{k}{L_{1}}\right)^{-1 / 6} C_{n}^{2}(h),
$$

where $L_{1}$ is the propagation distance in the free troposphere, $k$ denotes the optical wave number, and $k=2 \pi / \lambda$, here $\lambda$ is the optical wavelength. For the $\widetilde{C}_{n S}^{2}(h)$ profile in the stratosphere, this form is taken as suggested in [24],

$$
\widetilde{C}_{n S}^{2}(h)=13.75\left(\frac{k}{L_{2}}\right)^{2 / 3} C_{n}^{2}(h),
$$

where $L_{2}$ is the propagation distance in the stratosphere.

In addition, the outer scale changes with altitude, according to the empirical formula [26]

$$
L_{0}(h)=\frac{5}{1+\left(\frac{h-7500}{2500}\right)^{2}} .
$$

\section{Beam Wander}

The instantaneous center of the beam is randomly displaced in the receiver plane, producing what is commonly called the beam wander. Usually the phenomenon can be characterized statistically by the variance of the hot spot displacement along an axis or by the variance of the magnitude of the hot spot displacement [1]. Based on the Markov approximation, the beam wander or the variance of the instantaneous center of a Gaussianbeam wave in the receiver plane $(z=L)$ is modeled by the following expression

$$
\left\langle\rho_{c}^{2}\right\rangle=4 \pi^{2} k^{2} W^{2} \int_{0}^{L} \int_{0}^{\infty} \kappa \Phi_{n}(\kappa, z) H_{L S}(\kappa, z)\left(1-e^{\Lambda L \kappa^{2} \xi^{2} / k}\right) d \kappa d z
$$

where $\xi$ is the normalized distance variable, $W$ is the spot size radius at the receiver plane, $\Lambda$ is called the Fresnel ratio at the output plane, $\Phi_{n}(\kappa, z)$ represents the refractive-index fluctuation power spectrum, and $H_{L S}(\kappa, z)$ denotes the large-scale filter function [27]

$$
H_{L S}(\kappa, \xi)=\exp \left\{-\kappa^{2} W_{0}^{2}\left[\left(\Theta_{0}+\bar{\Theta}_{0} \xi\right)^{2}+\Lambda_{0}^{2}(1-\xi)^{2}\right]\right\}
$$

where $W_{0}$ denotes the radius of the beam size at the transmitter plane, $\Theta_{0}$ is also called the curvature parameter and $\Lambda_{0}$ is called the Fresnel ratio at the input plane, and $\bar{\Theta}_{0}=1-\Theta_{0}$.

Now, it is accepted that the influence of the atmospheric turbulence for the satelliteto-ground or ground-to-satellite link is a result of a combined action of the Kolmogorov turbulence in the boundary layer, non-Kolmogorov in the free troposphere, and nonKolmogorov in the stratosphere. Based on Reference [1], beam wander for a Gaussianbeam wave propagating through Kolmogorov turbulence in the boundary layer takes the following form

$$
\begin{aligned}
\left\langle\rho_{B c}^{2}\right\rangle & =7.25 L_{0}^{3} W_{0}^{-1 / 3} \int_{h_{0}}^{H_{1}} C_{n}^{2}(\xi) \xi^{2} \\
& \times\left\{\frac{1}{\left|\Theta_{0}+\bar{\Theta}_{0} \xi\right|^{1 / 3}}-\left[\frac{\kappa_{0}^{2}(h) W_{0}^{2}}{1+\kappa_{0}^{2}(h) W_{0}^{2}\left(\Theta_{0}+\bar{\Theta}_{0} \xi\right)^{2}}\right]^{1 / 6}\right\} d \xi,
\end{aligned}
$$

where $h_{0}$ is is the height of an optical transmitter/receiver above ground and $H_{1}$ is the altitude of the boundary layer. 
By following the approach in Reference [1], using Equation (5), beam wander a Gaussian-beam wave propagating through non-Kolmogorov turbulence in the free troposphere, is obtained (see Appendix A.1)

$$
\begin{aligned}
\left\langle\rho_{F \mathcal{C}}^{2}\right\rangle & =1.59 L_{1}^{3} W_{0}^{-2 / 3} \int_{H_{1}}^{H_{2}} C_{n F}^{2}(\xi) \xi^{2} \\
& \times\left\{\frac{1}{\left|\Theta_{0}+\bar{\Theta}_{0} \xi\right|^{2 / 3}}-\left[\frac{\kappa_{0}^{2}(h) W_{0}^{2}}{1+\kappa_{0}^{2}(h) W_{0}^{2}\left(\Theta_{0}+\bar{\Theta}_{0} \xi\right)^{2}}\right]^{1 / 3}\right\} d \xi .
\end{aligned}
$$

In the above equation, $\mathrm{H}_{2}$ is the altitude of the free troposphere.

In the same way, beam wander for a Gaussian-beam wave propagating through non-Kolmogorov turbulence in the stratosphere is also obtained (see Appendix A.2)

$$
\begin{aligned}
\left\langle\rho_{S c}^{2}\right\rangle & =0.34 L_{2}^{3} W_{0}^{-1 / 3} \int_{H_{2}}^{H} C_{n S}^{2}(\xi) \xi^{2} \\
& \times\left\{\left[\frac{\kappa_{0}^{2}(h) W_{0}^{2}}{1+\kappa_{0}^{2}(h) W_{0}^{2}\left(\Theta_{0}+\bar{\Theta}_{0} \xi\right)^{2}}\right]^{1 / 2}-\left|\Theta_{0}+\bar{\Theta}_{0} \xi\right|\right\} d \xi,
\end{aligned}
$$

where $H$ is the altitude of the satellite. Here, it is noteworthy that the the geometrical optics approximation is used in the derivation of Equations (15) and (16), viz.,

$$
1-e^{\Lambda L \kappa^{2} \xi^{2} / k} \simeq \frac{\Lambda L \kappa^{2} \xi^{2}}{k}, \quad L \kappa^{2} / k \ll 1 .
$$

In addition, to emphasize the refractive nature of beam wander, the last term in Equation (13) is also dropped.

According to Equation (7), it can be concluded that the beam wander displacement variance over the whole satellite-to-ground or ground-to-satellite link for small zenith angles or under weak irradiance fluctuations is expressed as the sum of the beam wander displacement variance caused by the turbulence in the boundary layer, the free troposphere, and the stratosphere.

$$
\begin{aligned}
\left\langle\rho_{c}^{2}\right\rangle & =\left\langle\rho_{B c}^{2}\right\rangle+\left\langle\rho_{F c}^{2}\right\rangle+\left\langle\rho_{S c}^{2}\right\rangle \\
& =7.25 L_{0}^{3} W_{0}^{-1 / 3} \int_{h_{0}}^{H_{1}} C_{n}^{2}(\xi) \xi^{2}\left\{\frac{1}{\left|\Theta_{0}+\bar{\Theta}_{0} \xi\right|^{1 / 3}}\right. \\
& \left.-\left[\frac{\kappa_{0}^{2}(h) W_{0}^{2}}{1+\kappa_{0}^{2}(h) W_{0}^{2}\left(\Theta_{0}+\bar{\Theta}_{0} \xi\right)^{2}}\right]^{1 / 6}\right\} d \xi \\
& +1.59 L_{1}^{3} W_{0}^{-2 / 3} \int_{H_{1}}^{H_{2}} C_{n F}^{2}(\xi) \xi^{2}\left\{\frac{1}{\left|\Theta_{0}+\bar{\Theta}_{0} \xi\right|^{2 / 3}}\right. \\
& \left.-\left[\frac{\kappa_{0}^{2}(h) W_{0}^{2}}{1+\kappa_{0}^{2}(h) W_{0}^{2}\left(\Theta_{0}+\bar{\Theta}_{0} \xi\right)^{2}}\right]^{1 / 3}\right\} d \xi \\
& +0.34 L_{2}^{3} W_{0} \int_{H_{2}}^{H} C_{n S}^{2}(\xi) \xi^{2}\left\{\left[\frac{\kappa_{0}^{2}(h) W_{0}^{2}}{1+\kappa_{0}^{2}(h) W_{0}^{2}\left(\Theta_{0}+\bar{\Theta}_{0} \xi\right)^{2}}\right]^{-1 / 2}\right. \\
& \left.-\left|\Theta_{0}+\bar{\Theta}_{0} \xi\right|\right\} d \xi .
\end{aligned}
$$

Here, it should be noted that beam wander is of little concern for a downlink path, but an uplink path can be large enough to cause the beam to move the several hundred meters. 
For a uplink path, the normalized variable $\xi$ has this form, $\xi=1-\left(h-h_{0}\right) /\left(H-h_{0}\right)$, and the beam wander displacement variance over the whole ground-to-satellite uplink for small zenith angles or under weak irradiance fluctuations is expressed as

$$
\begin{aligned}
\left\langle\rho_{c}^{2}\right\rangle & =\left\langle\rho_{B c}^{2}\right\rangle+\left\langle\rho_{F c}^{2}\right\rangle+\left\langle\rho_{S c}^{2}\right\rangle \\
& =7.25\left(H_{1}-h_{0}\right)^{2} \sec ^{3}(\zeta) W_{0}^{-1 / 3} \int_{h_{0}}^{H_{1}} C_{n}^{2}(h)\left(1-\frac{h-h_{0}}{H-h_{0}}\right)^{2} \\
& \times\left\{\frac{1}{\left|\Theta_{0}+\bar{\Theta}_{0}\left(1-\frac{h-h_{0}}{H-h_{0}}\right)\right|^{1 / 3}}-\left[\frac{\kappa_{0}^{2}(h) W_{0}^{2}}{1+\kappa_{0}^{2}(h) W_{0}^{2}\left(\Theta_{0}+\bar{\Theta}_{0}\left(1-\frac{h-h_{0}}{H-h_{0}}\right)\right)^{2}}\right]^{1 / 6}\right\} d h \\
& +1.59\left(H_{2}-H_{1}\right)^{2} \sec ^{3}(\zeta) W_{0}^{-2 / 3} \int_{H_{1}}^{H_{2}} C_{n F}^{2}(h)\left(1-\frac{h-h_{0}}{H-h_{0}}\right)^{2} \\
& \times\left\{\frac{1}{\left|\Theta_{0}+\bar{\Theta}_{0}\left(1-\frac{h-h_{0}}{H-h_{0}}\right)\right|^{2 / 3}}-\left[\frac{\kappa_{0}^{2}(h) W_{0}^{2}}{1+\kappa_{0}^{2}(h) W_{0}^{2}\left(\Theta_{0}+\bar{\Theta}_{0}\left(1-\frac{h-h_{0}}{H-h_{0}}\right)\right)^{2}}\right]^{1 / 3}\right\} d h \\
& +0.34\left(H-H_{2}\right)^{2} \sec ^{3}(\zeta) W_{0} \int_{H_{2}}^{H} C_{n S}^{2}(h)\left(1-\frac{h-h_{0}}{H-h_{0}}\right)^{2} \\
& \times\left\{\left[\frac{\kappa_{0}^{2}(h) W_{0}^{2}}{1+\kappa_{0}^{2}(h) W_{0}^{2}\left(\Theta_{0}+\bar{\Theta}_{0}\left(1-\frac{h-h_{0}}{H-h_{0}}\right)\right)^{2}}\right.\right. \\
& \left.\left.\left|\Theta_{0}+\bar{\Theta}_{0}\left(1-\frac{h-h_{0}}{H-h_{0}}\right)\right|\right\}^{-1 / 2} d h\right)
\end{aligned}
$$

where $\zeta$ is the zenith angle. It can be seen from Equations (15) and (16) that beam wander of a Gaussian-beam wave propagating through non-Kolmogorov atmospheric turbulence is independent on optical wave length, similar to Kolmogorov atmospheric turbulence [1].

\section{Simulation and Analysis}

For a satellite positioned in either a low Earth orbit (LEO) or a geostationary orbit (GEO) with the transmitter on the ground, beam wander displacement variance of a Gaussian-beam wave $\left(\Theta_{0}=1\right)$ is plotted as a function of beam radius at the receiver in Figure 1, based on the three-layer beam wander model Equation (19). The zenith angle is set $\zeta=0 \mathrm{deg}, C_{n}^{2}(0)=1.7 \times 10^{-15} \mathrm{~m}^{-2 / 3}$ and $h_{0}=0 \mathrm{~m}$ are taken, respectively. The outer scale model is described by Equation (11) with $\kappa_{0}(h)=2 \pi / L_{0}(h)$. And the theoretical model of conventional Kolmogorov beam wander displacement variance is also plotted in Figure 1 for comparison. Here, we can see that beam wander displacement variance is greatest for smaller beams and steadily decreases with an increasing beam radius. In addition, it can be seen from Figure 1 that the result predicted by the three-layer theoretical model is smaller than one predicted by the conventional Kolmogorov theory model, this is due to weaker fluctuations of non-Kolmogorov turbulence in the free troposphere and the stratosphere than Kolmogorov one, which matches the conclusions of Lukin et al. Beam wander displacement variance predicted by the three-layer theoretical model decreases sharply with the beam radius, with respect to the conventional Kolmogorov model.

Furthermore, beam wander displacement variance of a Gaussian-beam wave $\left(\Theta_{0}=1\right)$ for the three-layer model is plotted as the function of zenith angles in Figure 2 for $W_{0}=0.2 \mathrm{~m}$. As shown in Figure 2, beam wander displacement variance increases with the zenith angles, and increases lightly for smaller zenith angles and increases sharply for larger one.

Finally, in order to find the influence of the variations of nominal value of refractiveindex structure parameter on the ground $C_{n}^{2}(0)$ on beam wander, beam wander displacement variance of a Gaussian-beam wave $\left(\Theta_{0}=1\right)$ is plotted as a function of $C_{n}^{2}(0)$ in 
Figure 3, based on the three-layer beam wander model Equation (19). The zenith angle is set $\zeta=0$ deg and $W_{0}=0.2 \mathrm{~m}$ is taken. As shown in Figure 3, beam wander displacement variance increases with $C_{n}^{2}(0)$. Theoretically, the increase of refractive-index structure parameter indicates the enhancement of optical turbulence, which inevitably leads to the increase of beam wander.

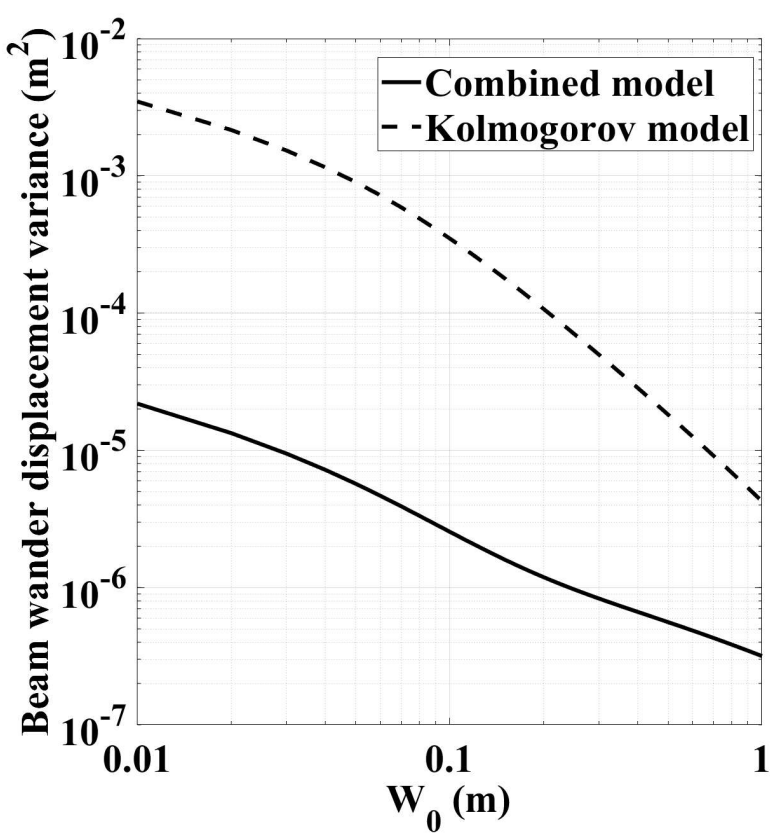

Figure 1. Beam wander displacement variance (solid curve) of a Gaussian-beam wave for the threelayer model plotted as a function of beam radius at the receiver for a transmitter on the ground and a satellite in space at zenith angle zero. The wavelength is $\lambda=1.55 \mu \mathrm{m}$ and the dashed curve corresponds to the conventional Kolmogorov model.

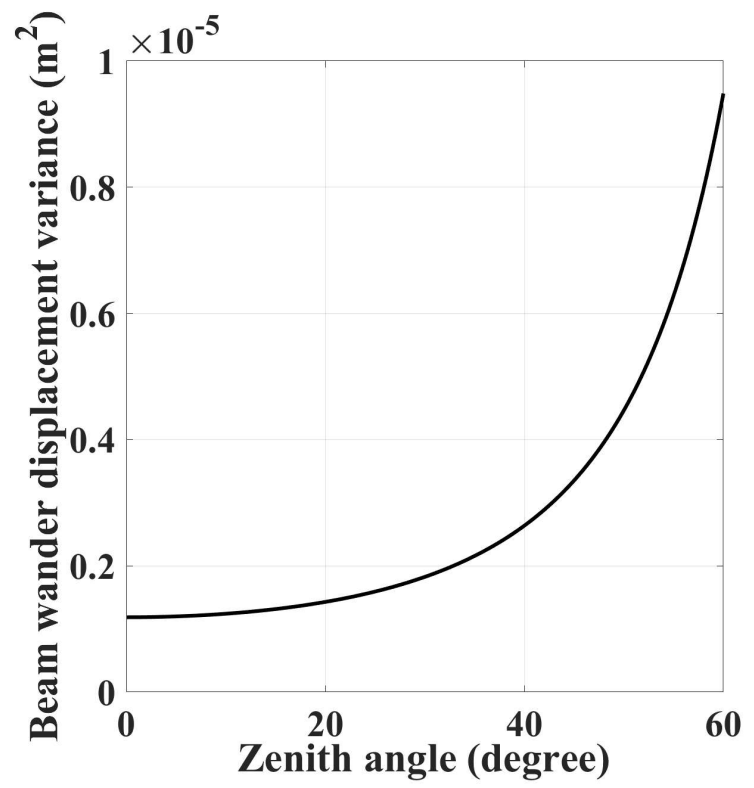

Figure 2. Beam wander displacement variance of a Gaussian-beam wave for the three-layer model plotted as a function of zenith angle for a transmitter on the ground and a satellite in space. 


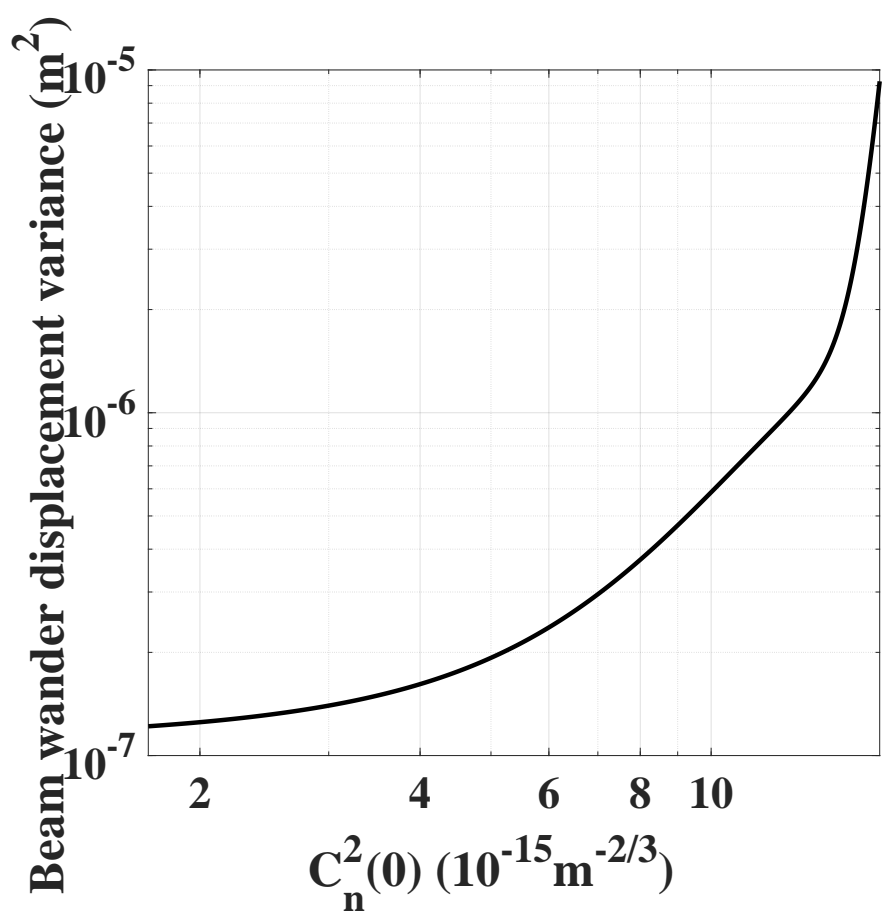

Figure 3. Beam wander displacement variance of a Gaussian-beam wave for the three-layer model plotted as a function of nominal value of refractive-index structure parameter on the ground $C_{n}^{2}(0)$ for a transmitter on the ground and a satellite in space.

\section{Conclusions}

In this paper, we proposed an exponential power spectra of refractive-index fluctuations for non-Kolmogorov turbulence in the free troposphere and the stratosphere, and on this basis, modified a three-layer altitude-dependent power spectrum of refractive-index fluctuations for satellite-to-ground and ground-to-satellite links, which is composed of the exponential Kolmogorov turbulence power spectrum of the boundary layer, the exponential non-Kolmogorov power spectrum turbulence of the free troposphere, and the exponential non-Kolmogorov power spectrum of the stratosphere. Based on the Markov approximation, beam wander displacement variances of a Gaussian-beam wave for non-Kolmogorov turbulence in the free troposphere and the stratosphere were derived, and the propagation theory of optical wave through atmospheric turbulence was further enriched. Further, the theoretical model of beam wander displacement variances of a Gaussian-beam wave propagating through Kolmogorov and non-Kolmogorov turbulence along laser-satellite communication uplink was developed and applied to characterize the displacement of the instantaneous center of the beam for the small zenith angle. Finally, the expression was used to analyze the variations of beam wander with beam radius, zenith angles, and a nominal value of refractive-index structure parameter on the ground.

Through our work in the paper, wander theoretical model of a Gaussian-beam wave propagating through Kolmogorov and non-Kolmogorov turbulence along laser-satellite communication uplink was established. According to the actual situation of the atmospheric turbulence along the satellite-ground link, the combined influence of Kolmogorov and non-Kolmogorov turbulence on beam wander was studied. It provides a theoretical basis for the design and practical application of a satellite-ground laser communication system. The research results not only further enrich the theory of atmospheric turbulent optical wave propagation, but also lay a theoretical foundation for the realization of high-speed satellite-ground laser communication, which has important guiding significance for the system design, in-orbit test, and practical application of satellite laser communication, and will further promote the development of satellite-ground laser communication technology. 
Author Contributions: Conceptualization, F.W. and W.D.; methodology, D.L., S.F. and W.D.; formal analysis, Q.Y.; investigation, F.W. and Q.Y.; data curation, Q.Y. and F.W., writing-original draft preparation, F.W. and W.D.; writing—review and editing, D.L., S.F., F.W. and W.D.; supervision, Q.Y. and W.D.; project administration, Q.Y. and W.D.; funding acquisition, Q.Y. and W.D. All authors have read and agreed to the published version of the manuscript.

Funding: This research was funded by the Foundation of Heilongjiang Educational Committee (FHEC) grant 12521603, the Natural Science Foundation of Heilongjiang Province of China (NSFHPC) grant LH2020F050, and the Scientific Research Project of Basic Scientific Research Funding for Universities in Heilongjiang Province (SRPBSRFUHP), grant 135309453 and 145109402.

Institutional Review Board Statement: Not applicable.

Informed Consent Statement: Not applicable.

Data Availability Statement: Data set available on request to corresponding authors.

Acknowledgments: We thank Ke Yang, Zheng Xu, and Jiahao Kang, who helped with the Matlab numerical simulation.

Conflicts of Interest: The authors declare no conflict of interest.

\section{Appendix A. Beam Wander a Gaussian-Beam Wave Propagating through Non-Kolmogorov Turbulence}

Appendix A.1. Beam Wander a Gaussian-Beam Wave Propagating through Non-Kolmogorov Turbulence in the Free Troposphere

For integration purposes, the normalized distance variable $\xi=1-z / L$ is used and Equation (12) becomes

$$
\left\langle\rho_{c}^{2}\right\rangle=4 \pi^{2} k^{2} W^{2} L \int_{0}^{1} \int_{0}^{\infty} \kappa \Phi_{n}(\kappa, \xi) H_{L S}(\kappa, \xi)\left(1-e^{\Lambda L \kappa^{2} \xi^{2} / k}\right) d \kappa d \xi
$$

The last term in the large-scale filter function Equation (13) is dropped to emphasize the refractive nature of beam wander, and then it is expressed as

$$
H_{L S}(\kappa, \xi)=\exp \left[-\kappa^{2} W_{0}^{2}\left(\Theta_{0}+\bar{\Theta}_{0} \xi\right)^{2}\right]
$$

In addition, here, the geometrical optics approximation is used and the last term in Equation (A1) becomes the following form,

$$
1-e^{\Lambda L \kappa^{2} \xi^{2} / k} \cong \frac{\Lambda L \kappa^{2} \xi^{2}}{k}, L \kappa^{2} / k \ll 1 .
$$

Substituting Equation (5), the power spectrum of non-Kolmogorov turbulence in the free troposphere, and Equation (A2) into Equation (A1) yields

$$
\begin{aligned}
\left\langle\rho_{c}^{2}\right\rangle & =0.592 k L^{2} W^{2} \Lambda \int_{0}^{1} \xi^{2} \widetilde{C}_{n F}^{2}(\xi) \int_{0}^{\infty} \kappa^{-1 / 3}\left[1-\exp \left(-\frac{\kappa^{2}}{\kappa_{0}^{2}(h)}\right)\right] \\
& \times \exp \left[-\kappa^{2} W_{0}^{2}\left(\Theta_{0}+\bar{\Theta}_{0} \xi\right)^{2}\right] d \kappa d \xi
\end{aligned}
$$

Using the integral identity

$$
\int_{0}^{\infty} \exp (-s t) t^{x-1} d t=\frac{\Gamma(x)}{s^{x}}
$$


and integrating the variable $\kappa$ in Equation (A4) yields

$$
\begin{aligned}
\left\langle\rho_{F c}^{2}\right\rangle & =1.59 L_{1}^{3} W_{0}^{-2 / 3} \int_{0}^{1} C_{n F}^{2}(\xi) \xi^{2} \\
& \times\left\{\frac{1}{\left|\Theta_{0}+\bar{\Theta}_{0} \xi\right|^{2 / 3}}-\left[\frac{\kappa_{0}^{2}(h) W_{0}^{2}}{1+\kappa_{0}^{2}(h) W_{0}^{2}\left(\Theta_{0}+\bar{\Theta}_{0} \xi\right)^{2}}\right]^{1 / 3}\right\} d \xi .
\end{aligned}
$$

Here it should be noted that the formula $\Lambda=2 L_{1} / \mathrm{kW}^{2}$ is used in the derivation.

Appendix A.2. Beam Wander a Gaussian-Beam Wave Propagating through Non-Kolmogorov Turbulence in the Stratosphere

Following Appendix A.1, beam wander for a Gaussian-beam wave propagating through non-Kolmogorov turbulence in the stratosphere is also obtained, namely Equation (16).

\section{References}

1. Andrews, L.C.; Philips, R.L. Laser Beam Propagation through Random Media; SPIE-The International Society for Optical Engineering Press: Bellingham, WA, USA, 2005. [CrossRef]

2. Lin, Z.; Lin, M.; Cola, T.; Wang, J.; Cheng, J. Supporting IoT With Rate-Splitting Multiple Access in Satellite and Aerial-Integrated Networks. IEEE Internet Things J. 2021, 8, 11123-11133. [CrossRef]

3. Lin, Z.; Lin, M.; Champagne, B.; Zhu, W.; Al-Dhahir, N. Secure and Energy Efficient Transmission for RSMA-Based Cognitive Satellite-Terrestrial Networks. IEEE Wirel. Commun. Lett. 2021, 10, 251-255. [CrossRef]

4. Lin, Z.; Lin, M.; Wang, J.; Cola, T.; Wang, J. Joint Beamforming and Power Allocation for Satellite-Terrestrial Integrated Networks With Non-Orthogonal Multiple Access. IEEE J. Sel. Top. Signal Process. 2019, 13, 657-670. [CrossRef]

5. Boroson, D.M.; Robinson, B.S.; Burianek, D.A.; Murphy, D.V.; Biswas, A. Overview and Results of the Lunar Laser Communication Demonstration. Proc. SPIE 2014, 8971, 89710S-1-89710S-11. [CrossRef]

6. Alonso, A.; Reyes, M.; Sodnik, Z. Performance of Satellite-to-ground Communications Link between ARTEMIS and the Optical Ground Station. Proc. SPIE 2004, 5572, 372-383. [CrossRef]

7. Wilson, K.E.; Kovalik, J.; Biswas, A.; Wright, M.; Roberts, W.T.; Takayama, Y.; Yamakawa, S. Preliminary Results of the OCTL to OICETS Optical Link Experiment (OTOOLE). Proc. SPIE 2010, 7587, 758703-1-758703-13. [CrossRef]

8. Toyoshima, M.; Takayama, Y.; Kunimori, H.; Jono, T.; Araib, K. Data Analysis Results from the KODEN Experiments. Proc. SPIE 2007, 7587, 67091C-1-67091C-12. [CrossRef]

9. Balin, S.; Yu, M.; Belen'kiy, S.; Mironov, V.L.; Samokhvalov, I.V.; Razenkov, I.A. Lidar Studis of the Aerosol Inhomogeneities in the Atmosphere. Atm. Ocean Opt. 1986, 22, 1060-1064.

10. Dalaudier, G.M.; Gurvich, A.S.; Kan, V.; Sidi, C. Middle Stratosphere Temperature Spectra Observed with Stellar Scintillation and in situ Techniques. Adv. Space Res. 1994, 14, 61-64. [CrossRef]

11. Zilberman, A.; Golbraikh, E.; Kopeika, N.S.; Virtser, A.; Kupershmidt, I.; Shtemler, Y. Lidar Study of Aerosol Turbulence Characteristics in the Troposphere: Kolmogorov and non-Kolmogorov Turbulence. Atmos. Res. 2008, 88, 66-77. [CrossRef]

12. Gurvich, A.S.; Kan, V. Structure of Air Density Irregularities in the Stratosphere from Spacecraft Observations of Stellar Scintillation: 1. Three-dimensional Spectrum Model and Recovery of Its Parameters. Izv. Atm. Ocean. Phys. 2003, 39, 300-310. [CrossRef]

13. Kyrazis, D.T.; Wissler, J.B.; Keating, D.D.B.; Preble, A.J.; Bishop, K.P. Measurement of Optical Turbulence in the Upper Troposphere and Lower Stratosphere. Proc. SPIE 1994, 2021,34-46. [CrossRef]

14. Gurvich, A.S. A Heuristic Model of Three-dimensional Spectra of Temperature Inhomogeneities in the Stably Stratified Atmosphere. Ann. Geophys. 1997, 15, 856-869. [CrossRef]

15. Sofieva, V.F.; Gurvich, A.S.; Dalaudier, F.; Kan, V. Reconstruction of Internal Gravity Wave and Turbulence Parameters in the Stratosphere Using GOMOS Scintillation Measurements. J. Geophys. Res. 2007, 112, 1-14. [CrossRef]

16. Dewan, E.M. Simulated Modelling of Internal Gravity Wave Spectra. Geophys. Res. Lett. 1991, 18, 1473-1476. [CrossRef]

17. Lukin, V.P.; Nosov, E.V.; Nosov, V.V.; Torgaev, A.V. Causes of Non-Kolmogorov Turbulence in the Atmosphere. Appl. Opt. 2016, 55, B163-B168. [CrossRef]

18. Nosov, V.V.; Kovadlob, P.G.; Lukin, V.P.; Torgaev, A.V. Atmospheric Coherent Turbulence. Atmos. Ocean. Opt. 2013, 26, 201-206. [CrossRef]

19. Lukin, V.P.; Bol'basova, L.A.; Nosov, V.V. Comparison of Kolmogorov's and Coherent Turbulence. Appl. Opt. 2014, 53, B231-B236. [CrossRef] [PubMed]

20. Du, W.; Zhu, H.; Liu, D.; Yao, Z.; Cai, C.; Du, X.; Ai, R. Influence of non-Kolmogorov Turbulence on Beam Spreading in Laser Satellite Communication. J. Russ. Laser Res. 2012, 33, 456-463. [CrossRef]

21. Du, W.; Yao, Z.; Liu, D.; Cai, C.; Du, X.; Ai, R. Influence of Non-Kolmogorov Turbulence on Intensity Fluctuations in Laser Satellite Communication. J. Russ. Laser Res. 2012, 33, 90-97. [CrossRef] 
22. Du, W.; Cheng, X.; Wang, Y.; Jin, Z.; Liu, D.; Feng, S.; Yang, Z. Scintillation Index of a Plane Wave Propagating through Kolmogorov and Non-Kolmogorov Turbulence along Laser-satellite Communication Downlink at Large Zenith Angles. J. Russ. Laser Res. 2020, 41, 616-627. [CrossRef]

23. Du, W.; Yuan, Q.; Cheng, X.; Wang, Y.; Jin, Z.; Liu, D.; Feng, S.; Yang, Z. Scintillation Index of a Spherical Wave Propagating Through Kolmogorov and Non-Kolmogorov Turbulence along Laser-Satellite Communication Uplink at Large Zenith Angles. J. Russ. Laser Res. 2021, 42, 150-161. [CrossRef]

24. Zilberman, A.; Golbraikh, E.; Kopeika, N.S. Propagation of Electromagnetic Waves in Kolmogorov and Non-Kolmogorov Atmospheric Turbulence: Three-layer Altitude Model. Appl. Opt. 2008, 47, 6385-6391. [CrossRef]

25. Mironov, V.L.; Nosov, V.V. On the Theory of Spatially Limited Light Beam Displacements in a Randomly Inhomogeneous Medium. J. Opt. Soc. Am. 1977, 67, 1073-1080. [CrossRef]

26. Coulman, C.E.; Vernin, J.; Coqueugniot, Y.; Caccia, J.L. Outer Scale of Turbulence Appropriate to Modeling Refractive-index Structure Profiles. Appl. Opt. 1988, 27, 155-160. [CrossRef]

27. Toselli, I.; Agrawal, B.; Restaino, S. Light Propagation through Anisotropic Turbulence. J. Opt. Soc. Am. A 2011, $28,483-488$. [CrossRef] [PubMed] 